\title{
Optimizing Pulsed OBIC Technique for ESD Defect Localization
}

\author{
Fabien Essely, Nicolas Guitard, Frédéric Darracq, Vincent Pouget, Member, IEEE, \\ Marise Bafleur, Senior Member, IEEE, Philippe Perdu, André Touboul, and Dean Lewis
}

\begin{abstract}
This paper presents a study of the well-known optical beam-induced current (OBIC) technique applied to electrostaticdischarge defect localization. The OBIC technique is improved by using a pulsed laser beam instead of a continuous one. Critical parameters of the experimentation are explored in this paper. We particularly discuss on the influence of the laser energy, the bias of the device under test and the spatial resolution of the technique.
\end{abstract}

Index Terms-Electrostatic discharge (ESD) defect, influence of experimental parameter, pulsed optical beam-induced current (OBIC).

\section{INTRODUCTION}

$\mathbf{T}$ HE EVOLUTION of laser sources has led to the advent of new laser-based techniques for failure analysis. The pulsed optical beam-induced current (OBIC) technique [1], [2], [4] is one of them, which is based on the photoelectric laser stimulation of the device under test (DUT) at a micrometric scale. The suitability of this technique to localize failure sites resulting from electrostatic discharges (ESDs) has previously been demonstrated [2]. The properties of pulsed OBIC technique compared with other existing localization techniques are discussed in another work. The details of this work could be found in [3]. This paper presents a complementary work on the OBIC experimental procedure for defect localization. It aims at improving the sensitivity of this technique and, thus, the probability to localize very small-size defects.

\section{EXPERIMENTAL SETUP}

A thorough description of the experimental laser test bench developed at IXL laboratory can be found in [5]. The laser source is a Ti:sapphire laser delivering picosecond pulses at an 82-MHz frequency (see Fig. 1).

A pulse picker allows the control of this frequency from several megahertz to a single shot (see Fig. 1). The laser

Manuscript received September 21, 2006; revised July 26, 2007.

F. Essely is with the Central Characterization and Analysis Laboratory, STMicroelectronics, 13106 Rousset, France (e-mail: essely@ixl.fr).

$\mathrm{N}$. Guitard is with the NXP Semiconductors, 6534 AE Nijmegen, The Netherlands.

F. Darracq, V. Pouget, A. Touboul, and D. Lewis are with the Laboratoire de l'Intégration du Matériau au Systéme, Université de Bordeaux 1, 33405 Talence, France.

M. Bafleur is with the Laboratoire d'Analyse et d'Architecture des Systèmes, Centre National de la Recherche Scientifique, 31077 Toulouse, France.

P. Perdu is with the Centre National d'Etudes Spatiales, 31401 Toulouse, France.

Color versions of one or more of the figures in this paper are available online at http://ieeexplore.iee.org.

Digital Object Identifier 10.1109/TDMR.2007.911381

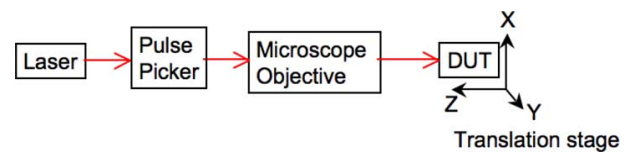

Fig. 1. Optical part of the OBIC experimental test bench.

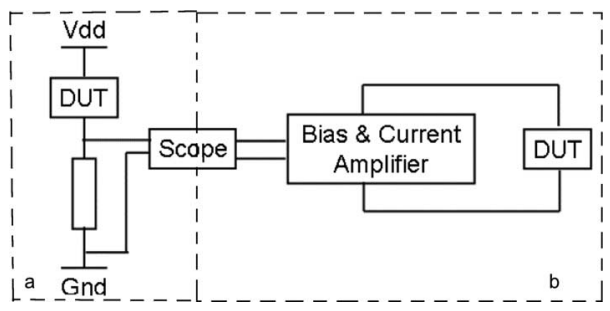

Fig. 2. Electrical part of the OBIC experimental test bench.

wavelength is tunable from 780 to $1000 \mathrm{~nm}$. For these studies, the wavelength is set to $800 \mathrm{~nm}$. The laser beam is focused by a $100 \times$ microscope objective to obtain a laser spot on the DUT of $1 \mu \mathrm{m}$ diameter. To provide a mapping of the OBIC signal, the DUT is mounted on an XYZ translation stage whose minimum step is $0.1 \mu \mathrm{m}$. This step is lower than the laser-spot diameter. We will discuss in the next part the impact of this step size on the OBIC spatial resolution.

During the test, the IC is biased, and the photocurrent measurement is done through a resistor connected between the IC's ground and the ground of the power supply [see Fig. 2(a)]. On some samples, the amplitude of the photocurrent is lower than the amplitude of the noise of the electrical setup. Thus, we have to use a current amplifier to bias the DUT [see Fig. 2(b)].

\section{Devices Under Study}

The first device is a test circuit consisting in a CMOS inverter chain designed in a $0.6 \mu \mathrm{m}$ technology. One of these inverters has a separated power supply access which is not ESD-protected. It permits the application of ESD stresses only on this inverter to induce defects into the circuit core [7]. The quiescent current measurement was the chosen failure indicator. We applied ten cumulative ESD stresses according to the socketed charged-device model of $2 \mathrm{kV}$ on every sample. Only the inverter with the independent supply access has been stressed; it is shown on Fig. 3.

Table I indicates the increase of the quiescent current for the three stressed devices. The different evolution of the quiescent current suggests the creation of different defects in each of the three inverters. 


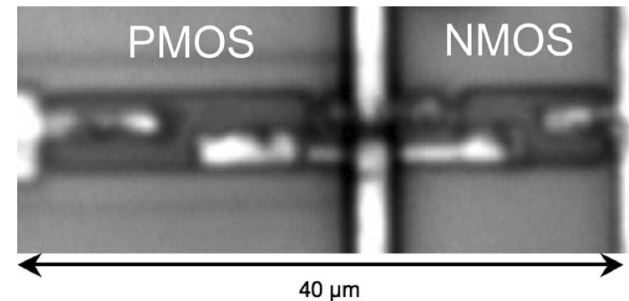

Fig. 3. Stressed inverter.

TABLE I

EVOLUTION OF THE QUIESCENT CURRENT OF THE STRESSED INVERTERS

\begin{tabular}{|c|c|c|c|}
\hline Sample & INV2 & INV5 & INV9 \\
\hline Current increase & $+30 \mathrm{nA}$ & $+50 \mu \mathrm{A}$ & $+1.5 \mu \mathrm{A}$ \\
\hline
\end{tabular}

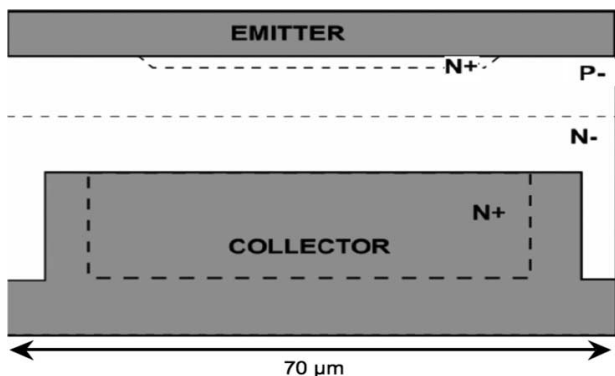

Fig. 4. Schematic view of the tested GBNPN.

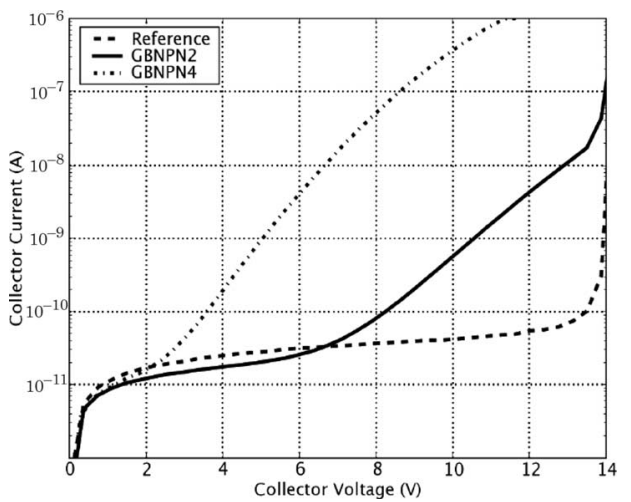

Fig. 5. Electrical characteristic of a reference IC and of the two stressed ICs.

The second kind of device is a grounded-base n-p-n (GBNPN) transistor with a graded $\mathrm{N}+/ \mathrm{N}$ collector used as an ESD protection. A schematic view of this structure is shown in Fig. 4.

The static $I(V)$ curve is the chosen failure criterion. Fig. 5 shows the electrical characteristic of an unstressed GBNPN and two stressed devices. In static polarization, the behavior of this device is similar to a reverse-biased diode. The device GBNPN4 has been stressed four times according to the human-body model (HBM) at $4 \mathrm{kV}$. The other component (GBNPN2) has been stressed according to the transmission-line-pulse (TLP) model. We applied multiple stresses in order to create potentially different kinds of ESD defects. The increase of the collector current indicates the creation of different defects in both samples.

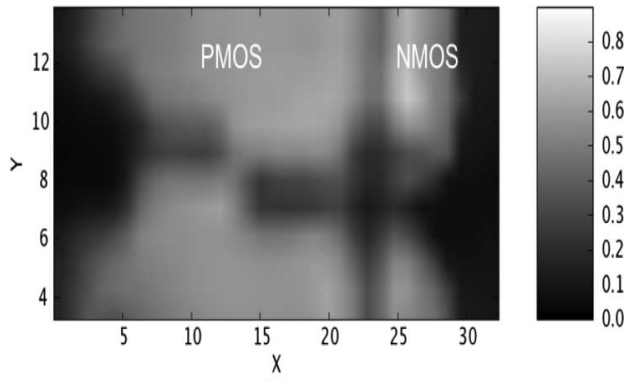

(a)

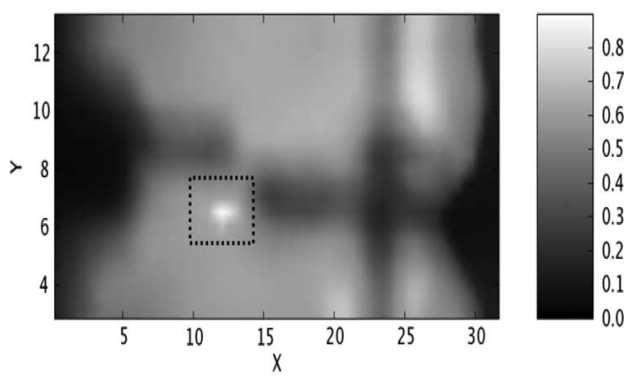

(b)

Fig. 6. OBIC images of the two inverters. (a) Reference device. (b) ESD stressed device.

\section{EXPERIMENTAL RESUlTS}

Fig. 6 shows the maps obtained for two inverter chain circuits: one stressed (b) and the other unstressed used as reference (a). The displayed parameter is the maximum value of the OBIC, which is displayed in arbitrary unit by the color scale of Fig. 6. It is normalized by the maximum value of the two maps. Any differences of more than $20 \%$ between the reference map and the map of the stressed device are interpreted as the presence of a failure site. In Fig. 6, the $X$ - and $Y$-axes are given in micrometers.

The cartography of Fig. 6 corresponds to the inverter shown in Fig. 3. In Fig. 6(b), the dotted area corresponds to a higher amplitude of the photocurrent (33\%) than in the reference device [see Fig. 6(a)]. This area pinpoints a defect clearly located in the PMOS transistor and expected to be a melted silicon filament that partially shunts the drain substrate junction.

Fig. 7 shows the maps obtained by the OBIC techniques for the GBNPN samples. The measured parameter is the maximum of the photocurrent. The $X$ and $Y$-axes are in micrometers, and the color scale is normalized by the maximum of the three maps. The strong increase (about $40 \%$ ) of the signal, in the white dotted frames in Fig. 7(b) and (c), pinpoints the defect localization in the two tested samples. In both cases, the defects are located in the collector area.

Two defects are localized in GBNPN4 but only one in GBNPN2. This is due to the different stress methods applied on the samples (HBM for GBNPN4 and TLP for GBNPN2). More explanations can be found in [10].

\section{A. Spatial Resolution}

To study the influence of the displacement step value on the OBIC spatial resolution, an IC is mapped at constant laser energy and for three different displacement step values. The 


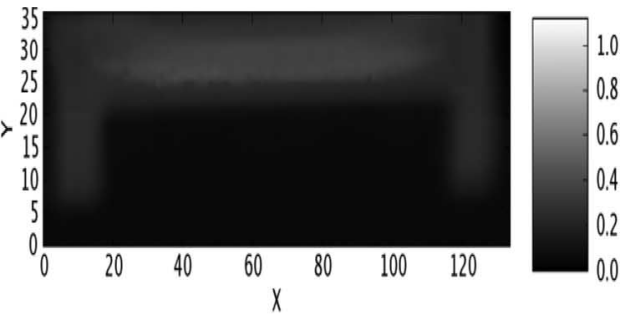

(a)

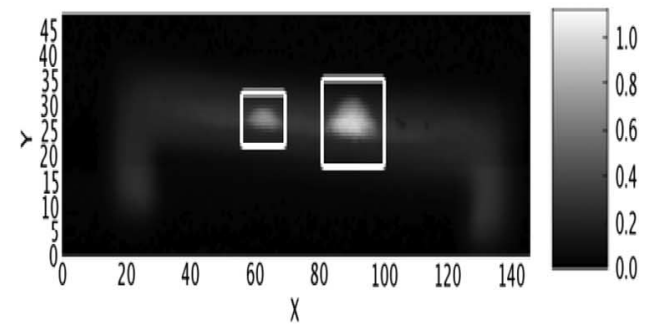

(b)

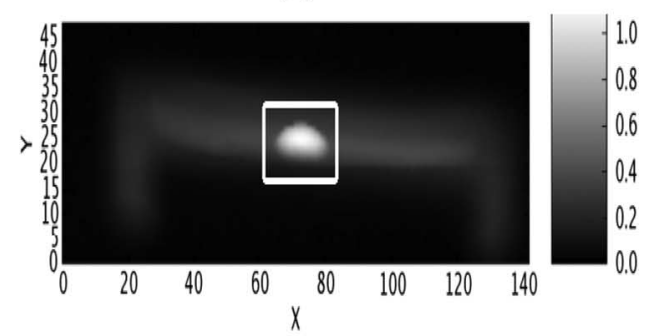

(c)

Fig. 7. OBIC images of three GBNPNs. (a) Reference device. (b) GBNPN4. (c) GBNPN2.

TABLE II

Apparent Area (IN SQuare Micrometers) of the Defect For THREe VAlues of THE DisPlacement STEP

\begin{tabular}{|c|c|c|c|}
\hline Sample/Step $(\mu \mathrm{m})$ & 0.2 & 0.5 & 1 \\
\hline INV2 & 1.48 & 1.75 & 3 \\
\hline INV5 & 1.75 & 3.50 & Undetected \\
\hline INV9 & 7.80 & 9.75 & 11 \\
\hline
\end{tabular}

area of the detected defective region is presented in Table II for three different inverter chain circuits and three values of the displacement step.

The results in Table II show a significant influence of the displacement step on the spatial resolution of the technique. To perform a complete failure analysis, physical deprocessing or cross-sectioning is generally carried out and requires a knowledge, as precisely as possible, of the defect location. Because of their small size, some defects cannot be optically observed. A scanning electron microscope or a transmission electron microscope is therefore necessary to observe it. However, as the field of view is strongly reduced, the preliminary localization of the defect has to be as precise as possible. The results on samples INV2, INV5, and INV9 underline the dependence between the apparent size of the defect and the displacement step. By decreasing the displacement step, we observe that the size of the defect is also decreasing for INV2 and INV9. The smaller is the size of the defect, the better it is to perform the physical analysis. However, the duration of the experimentation should be taken into account. To obtain the best resolution, the

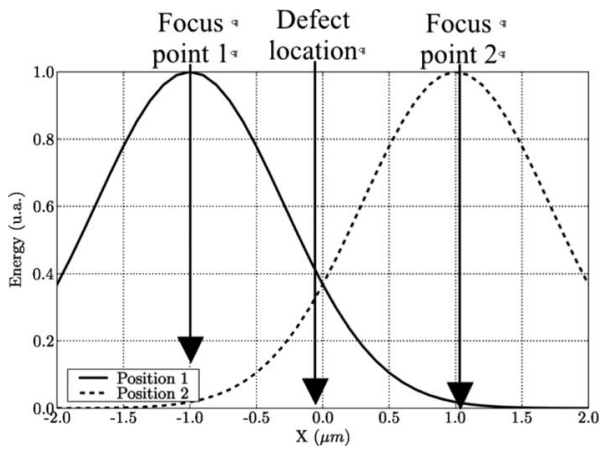

Fig. 8. Laser energy deposited on two positions.

duration of the experimentation is indeed strongly increased. For example, by dividing the displacement step by ten, we multiply the duration of the experimentation by 100 . Therefore, the experimenter should find a compromise between the apparent size of the defect and the duration of the OBIC test.

INV5 is an interesting case since it shows that, for the largest step value $(1 \mu \mathrm{m})$, the defect does not appear in the mapping. Nonetheless, it probably would for higher pulse energies. We will discuss in the next section the influence of the sensibility to latchup. Our hypothesis is based on the spatial repartition of the laser pulse energy deposited on the IC. It has a Gaussian shape with an $1 \mu \mathrm{m}$ diameter. Thus, the energy is quickly radially decreasing. On studying INV5 with a $1 \mu \mathrm{m}$ step, we may not focus the laser exactly on the defect but a little around it. Fig. 8 shows the Gaussian shape of the laser beam and the two focus points around the defect. Therefore, the major part of the pulse energy does not contribute to the defect activation.

As a consequence, we think that, by increasing the laser energy, we would be able to locate the defect with a $1 \mu \mathrm{m}$ placement step. Indeed, increasing the total energy of the laser pulse implies to have more energy on the side of the Gaussian, i.e., on the defect site.

The amount of laser energy has also an influence on the spatial resolution. By increasing this energy, we increase the possibility to activate the defect even if the laser is focused far from the defect. Thus, the spatial localization is less accurate with high laser energy than with low laser energy.

In the next part, we will see the influence of laser pulse energy in defect localization.

\section{B. Influence of the Bias Voltage}

Fig. 9 shows the variation of the OBIC versus the bias of the GBNPN2. The laser energy has been chosen as high as required for not being a limitation in the defect detection. This way, only the bias condition could limit the defect localization capability.

We note that Fig. 5 corresponds to the electrical characteristic of the tested device. Fig. 5 shows that the failure begins to be observed at a bias value of $7 \mathrm{~V}$. This means that the defect is electrically activated above this bias value. Fig. 9 shows the maximum of the photocurrent on two devices with regard to the bias voltage. This permits us to study the impact of the bias voltage on our capability to detect a defect. As shown in Fig. 9, no significant increase of the photocurrent (comparatively to the reference device) is observed before the electrical activation of 


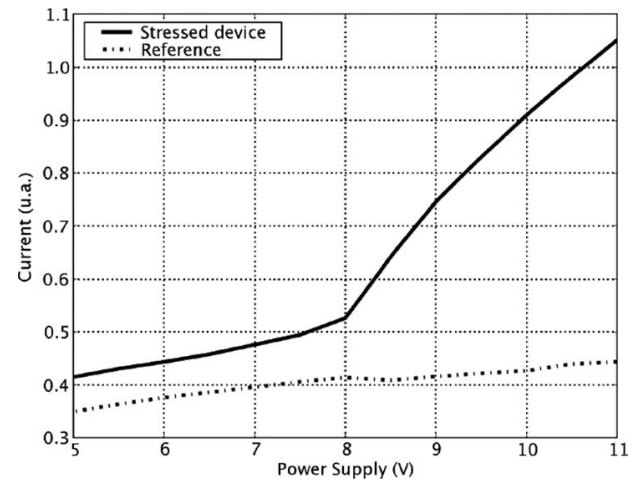

Fig. 9. OBIC versus bias voltage of GBNPN2.

the defect. As a result, the defect cannot be localized with a biased voltage below $8 \mathrm{~V}$. Previous studies have shown that the increase of the photocurrent due to the defect is due to an avalanche process caused by the shape of the defect itself [2]. This means that, in biased OBIC technique, the defect has to be electrically activated to be visible. This electrical activation corresponds to the one seen in Fig. 5 because the physical phenomenon in charge is the same. Nevertheless, we notice a difference between the two mentioned voltages. This underlines that the avalanche process has to be stronger in the OBIC technique than in the $I(V)$ measurement to detect a difference.

\section{Energy Mapping}

In this section, we study the influence of the laser energy on OBIC defect localization. The curves shown in Fig. 10(a) have been obtained during the test of the two types of DUT. Each curve is the difference between the maximal current measured on the defect $\left(I_{\mathrm{d}}\right)$ and on the same point on the reference $\left(I_{\mathrm{r}}\right)$. $I_{\mathrm{d}}-I_{\mathrm{r}}$ pinpoints clearly the influence of the laser energy on the defect detection. Both of them [see Fig. 10(a)] indicate the presence of an energy threshold value for which a significant relative increase of the current is observed. This energy depends on the sample type and on the defect. The measured energy threshold is around $40 \mathrm{pJ}$ for the inverter and around $100 \mathrm{pJ}$ for the GBNPN. This behavior can be interpreted as a threshold effect, but to date, the origin of this threshold is not fully understood. Indeed, a moderate variation of the laser pulse energy induces a slight difference in the number of generated carriers. However, the observed current depends only on the collection processes of these carriers. As shown in the previous section, avalanche process is involved in the collection. Thus, such effect could justify the optical threshold in terms of the trigger of an amplification of the collected current.

Fig. 10(b) shows the relationship between the threshold detection and the bias of the device. The power supply voltage is relevant for the electrical defect activation. Fig. 10(b) shows that, by decreasing the electrical defect activation, OBIC technique requires an increase of the laser energy to be able to detect the defects. The best compromise on the choice of the laser energy and the bias voltage depends on the IC and the defect nature and shape. It is then impossible to predict what would be the best values before beginning the test procedure. The bench scientist should take care of some other phenomena

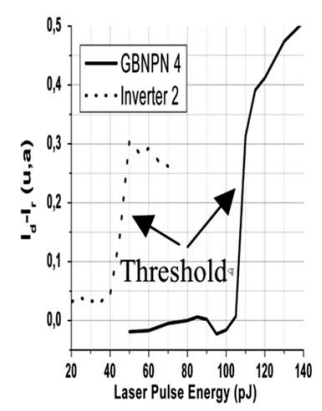

(a)

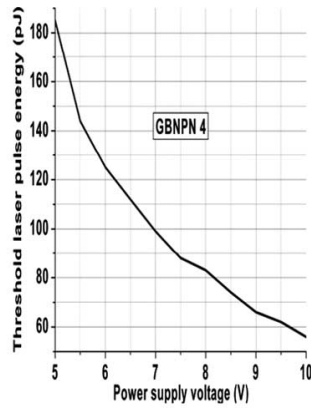

(b)
Fig. 10. (a) Amplitude of OBIC versus pulse laser energy. (b) Energy threshold versus power supply.

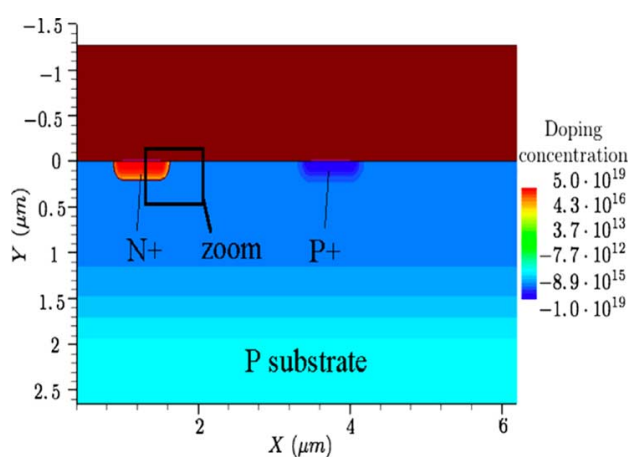

Fig. 11. Doping concentration of the device used in physical simulation.

such as latchup. Indeed, by applying a standard bias voltage on the DUT, the laser pulse could trigger a latchup, which could be destructive for the device [6]. In our case, the inverters have proven to be latchup-sensitive during the OBIC test. Thus, in our experiments on these devices, we have reached this compromise with a laser pulse energy of $50 \mathrm{pJ}$ for a bias voltage of $4.5 \mathrm{~V}$.

In order to understand the threshold behavior of Fig. 10(a), we carried out physical simulation with an ISE-TCAD tool suite [8].

\section{Physical Simulations}

In order to understand the laser-energy threshold effect on the OBIC defect localization presented in this paper, physical simulations have been brought into play.

\section{A. Definition of the Simulated Devices}

The modeled device is a diode shown in Fig. 11. This device has been chosen because it is the common structure responsible for the defect creation in each of the tested devices [10]. The doping concentration values correspond to the technological data of the GBNPN's technology. The two types of tested devices have not been simulated because all the needed technological data are not available.

The zoom area corresponds to the studied region in the next part. To compare the simulation results with the experimental ones, we have to do simulations on a stressed device and on a reference. In the first step, we have to introduce a defect in the 


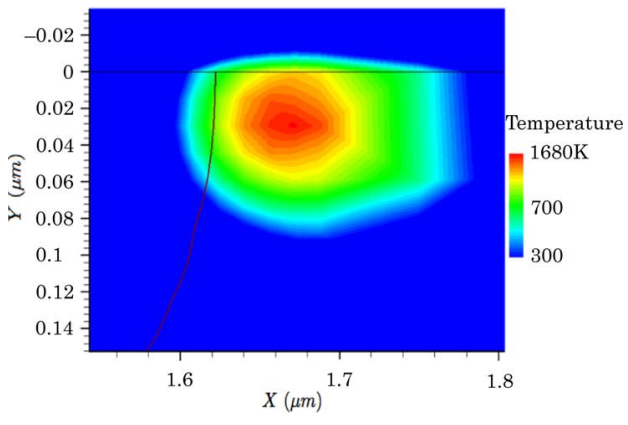

Fig. 12. Hot-spot localization during a 500-V TLP stress.

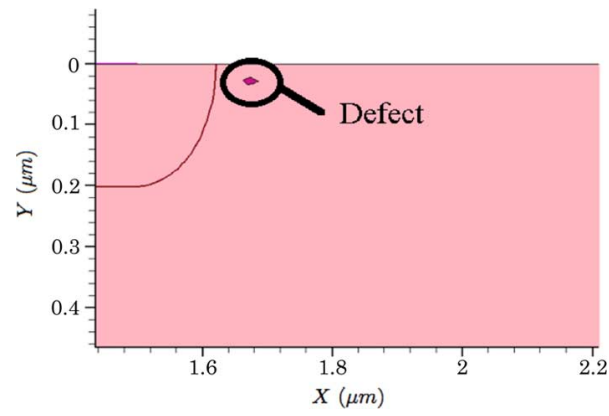

Fig. 13. Modeled defect in the simulated diode.

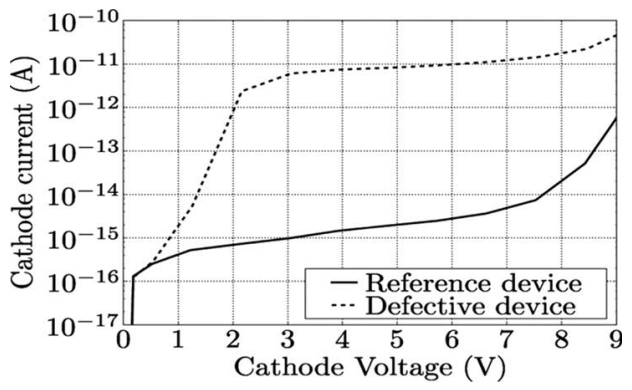

Fig. 14. Electrical behavior of the reference device and the device with a modeled defect.

device. The previous experimentations reveal only bulk defect. This kind of defect is only composed of melted silicon.

In order to determine the position and the size of the defect, we carried out physical simulation involving a TLP pulse on the reference structure (see Fig. 11). The chosen stress amplitude is $500 \mathrm{~V}$.

Fig. 12 shows the heat generated during this stress and before the melting point of silicon is reached. The position of the highest heat increase corresponds to the probable position of the defect. The heat extension corresponds to the size of the defect.

The previous simulated result allows designing the ESD defect shown in Fig. 13. It is composed of a polysilicon which is the closest material, available in the simulator, to the melted silicon.

Fig. 14 shows the evolution of the electrical characteristic of the device with and without introducing the modeled defect. We notice a strong increase of the quiescent current when the device is biased with a voltage above $1 \mathrm{~V}$. This behavior corresponds qualitatively to the result obtained on the GBNPN but not quantitatively because of the differences between the devices. In order to understand the phenomena involved during

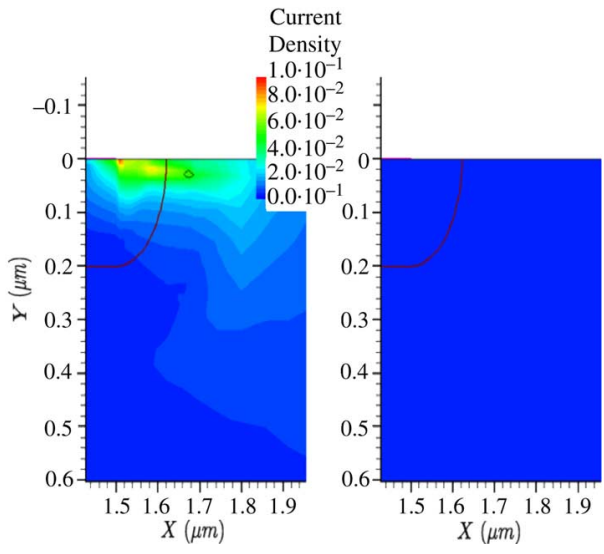

Fig. 15. Comparison between the current density (in amperes per cubic centimeter) of (left) the defective device and (right) the reference one.

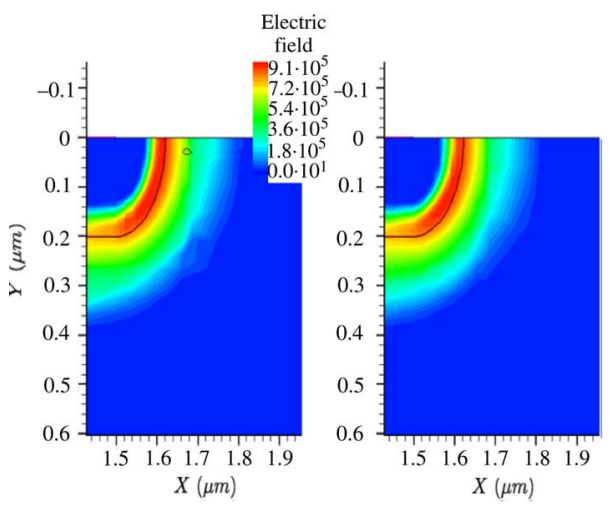

Fig. 16. Comparison between the electric field (in volts per centimeter) of (left) the defective device and (right) the reference one.

the OBIC technique, we first have to pinpoint the mechanisms involved in the modification of the electrical characteristic.

The increase of the quiescent current implies a variation on the current density in the device. Fig. 15 shows the variation due to the defect for a reverse-bias voltage of $9 \mathrm{~V}$.

The increase of the current density is strongly correlated with the defect position. Indeed, most of the current goes through the defect. Fig. 16 shows the electric field in the two devices with the same bias condition. Surprisingly, the contour plots of the electric field inside the defective and the reference device are similar. Thus, the increase of the quiescent current is due to another phenomenon.

The impact ionization appearing in the two devices at a bias voltage of $9 \mathrm{~V}$ is shown in Fig. 17. We could notice a strong difference between these two plots. This phenomenon is located exactly at the left end of the defect.

We can conclude that this phenomenon implies the increase of the quiescent current.

\section{B. Influence of the Laser Energy}

The difference of the maximum value of the photocurrent simulated on the defective and on the reference device, which is shown in Fig. 18, is a function of the laser pulse energy. The simulations have been done for three bias-voltage values: 1,5 , and $9 \mathrm{~V}$. The curves of Fig. 18 show the relationship between this difference and the laser pulse energy. 


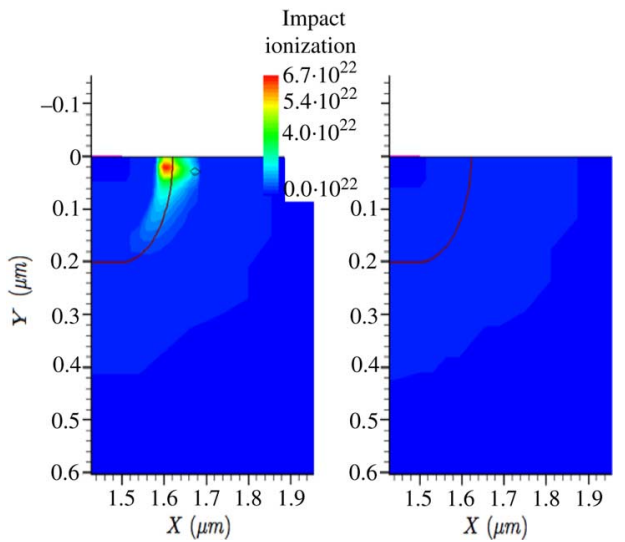

Fig. 17. Comparison between the impact ionization (per second per cubic centimeter) of (left) the defective device and (right) the reference one.

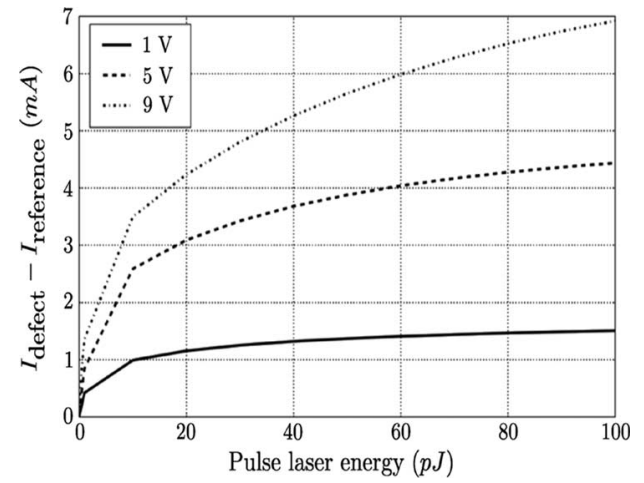

Fig. 18. Influence of the laser pulse energy on the difference between the maximum of the photocurrent of a defective device and the same on the reference device for three bias voltages.

The threshold behavior observed in the experimentation is not reproduced in simulation, but we can notice that the difference between the maximum of the photocurrents increases with the laser energy. This trend is thus roughly similar to the one observed in the experimentation.

In order to understand the phenomena involved in this paper, the electric field is shown in Fig. 19. We could notice some differences on its behavior. The variation of the laser pulse energy from $50 \mathrm{fJ}$ to $90 \mathrm{pJ}$ is sufficient to imply a different electrical-carrier injection level. For $50 \mathrm{fJ}$, the injection level is low. No modification of the electric field is observed during the laser excitation [see Fig. 19(a)]. Fig. 19(b) shows the variation of the electric field for a laser energy of $90 \mathrm{pJ}$.

Thus. we suppose that the increase of the photocurrent in the defective device is strongly correlated to the modification of the electric field.

\section{Influence of the Bias Voltage on the OBIC Response of the DUT}

The parameters (pulse duration, wavelength, spot size, etc.) of the simulated beam are similar to the experimental ones. Fig. 20 shows the difference between the maximum of the photocurrent obtained with the defective device and the maximum of the photocurrent obtained with the reference device with regard to the bias voltage. This result is obtained with a laser pulse energy of $90 \mathrm{pJ}$. The photocurrent is always greater than
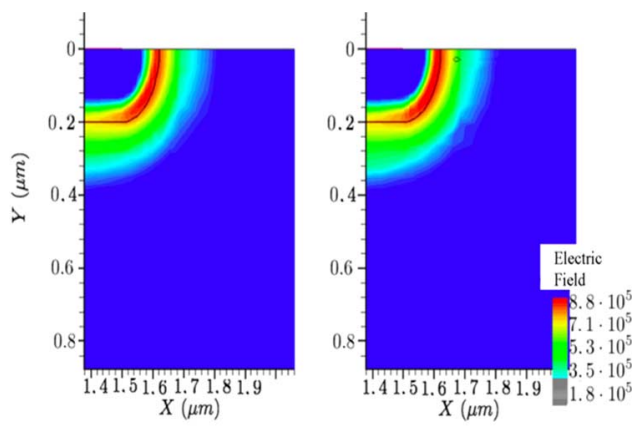

(a)
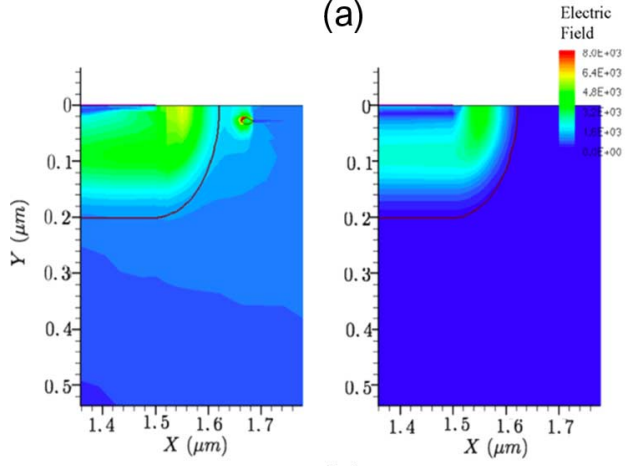

(b)

Fig. 19. Behavior difference on the electric field during the laser excitation. (a) Energy of 50 fJ. (b) Energy of 90 pJ.

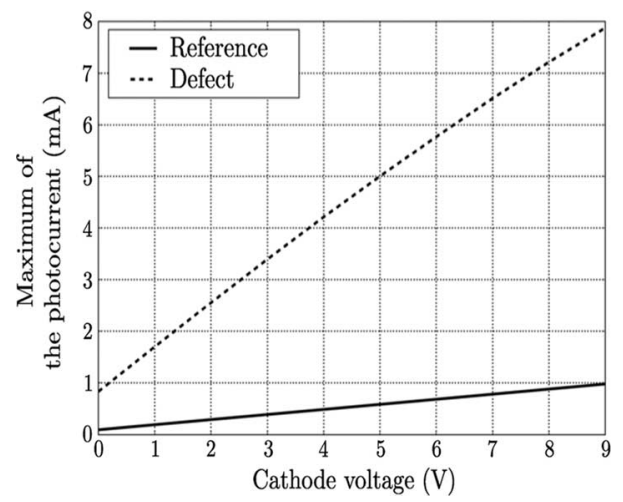

Fig. 20. Influence of the bias voltage on the maximum of the photocurrent.

the photocurrent obtained on the reference device. In order to understand the phenomenon involved in this curve, let us just be reminded of the way the photocurrent is created [1]. It is composed of two parts: a drift current and a diffusion current. The electrical carriers generated in an electric field are quickly separated, and they create the drift current. The electric field could be created by a simple p-n junction or by an external bias. When the electrical carriers are generated in a region without an electric field, they could simply recombine themselves. The other phenomenon is the apparition of an ambipolar diffusion, which gives the diffusion current. It is slower than the drift current. In this case, the maximum of the photocurrent is due to the drift contribution. However, we do not notice any differences on the electric field for the two devices before the laser pulse's arrival (see Fig. 16). By focusing a laser beam on a DUT, the generated electrical-carrier density can disturb the electric field due to the bias [11]. Fig. 19(b) shows the electric filed in the two devices at the maximum power of the laser pulse. 
A significant difference is observed on these curves. The spatial extension of the electric field in the defective device is more important than in the reference device. A peak of the electric field is observed at the left end of the defect. This behavior is similar at any bias voltage for a high enough laser pulse energy. This difference in the electric field explains the increase of the photocurrent.

\section{CONCLUSION}

This paper gives some insights on the critical parameters involved in the detection of ESD defects using pulsed-laser OBIC technique. It appears that bias voltage and laser energy are important and strongly correlated. On the other side, the spatial resolution has to be taken carefully into account. Simulation models must be improved to precisely understand the threshold mechanisms observed in these OBIC experiments.

\section{ACKNOWLEDGMENT}

The authors would like to thank the region Aquitaine for its support.

\section{REFERENCES}

[1] T. Wilson et al., "Theory of optical beam induced current images of defects in semiconductors," J. Appl. Phys., vol. 61, no. 1, pp. 191-195, Jan. 1987.

[2] T. Beauchne et al., "ESD defect localisation using photovoltaic laser stimulation techniques: Optimization and interpretation," in Proc. IPFA, 2003, pp. 179-182.

[3] F. Essely et al., "Application of various optical techniques for ESD defect localization," Microelectron. Reliab., vol. 46, no. 9-11, pp. 1563-1568, Sep.-Nov. 2006.

[4] J. Fritz et al., "Techniques for picosecond OBIC measurement on ICs," Microelectron. Eng., 1990, 12, 381.

[5] V. Pouget et al., "Time-resolved scanning of integrated circuits with a pulsed laser: Application to transient fault injection in an ADC," in Proc. IMTC, 2003, pp. 1376-1380.

[6] P. Fouillat et al., "Localization and characterization of latch-up sensitive areas using a laser beam: Influence on design rules of ICs in CMOS technology," Qual. Reliab. Eng. Int., vol. 9, no. 6, pp. 477-482, 1993.

[7] N. Guitard et al., "ESD induced latent defects in CMOS ICs and reliability impact," in Proc. EOS/ESD Symp., 2004.

[8] Manuals of ISE-TCAD.

[9] F. Beaudoin, "Localisation de défaut par la face arriére des circuits intégrés," Ph.D. dissertation, Univ. Bordeaux 1, Bordeaux, France, 2002.

[10] N. Guitard et al., "Different failure signatures of multiple TLP and HBM stresses in an ESD robust protection structure," Microelectron. Reliab., vol. 45, no. 9-11, pp. 1415-1420, 2005.

[11] C. M. Hsieh et al., "A field-funneling effect on the collection of alphaparticle-generated carriers in silicon devices," IEEE Electron Device Lett., vol. EDL-2, no. 4, pp. 103-105, Apr. 1981

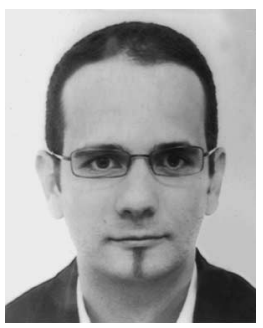

Fabien Essely was born in 1979. He received the $\mathrm{Ph} . \mathrm{D}$. degree in electronics from Bordeaux University, Bordeaux, France, in 2006. He studied methodologies of failure analysis, and particularly techniques using a pulsed laser beam, in collaboration with Dean Lewis.

He is currently a Postdoctoral Appointee with the Central Characterization and Analysis Laboratory, STMicroelectronics, Rousset, France.

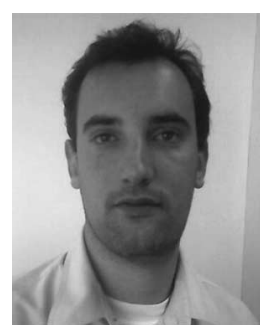

discharge stress.

$\mathrm{He}$ is currently with the NXP Semiconductors (formerly Philips Semiconductors), Nijmegen, The Netherlands as an ESD/LU Engineer.

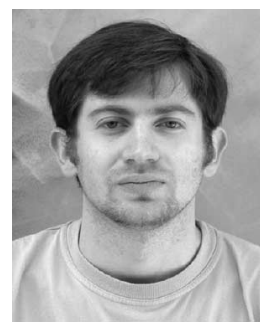

Frédéric Darracq was born in 1974. He received the $\mathrm{Ph} . \mathrm{D}$. degree in electronics from Bordeaux University, Talence, France, in 2003.

$\mathrm{He}$ is currently an Associate Professor with the Université de Bordeaux 1, Talence, teaching electronics, where he is with the Laboratoire de l'Intégration du Matériau au Systéme (IMS Laboratory). His research activities at the IMS Laboratory consist of the use of ultrashort laser pulses to test the sensitivity of integrated circuits to some effects induced by ionizing particles.

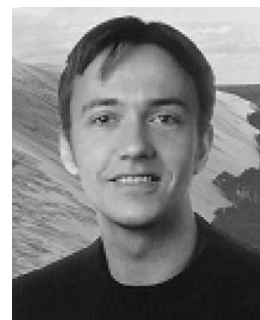

Vincent Pouget (M'03) received the Ph.D. degree in instrumentation and measurement from the Université de Bordeaux 1, Bordeaux, France, in 2000.

$\mathrm{He}$ is currently a Full-Time Research Scientist with the Laboratoire de l'Intégration du Matériau au Systéme, Université de Bordeaux 1, Talence. His main research interests include pulsed-laser testing and analysis of integrated circuits and radiation effects in embedded systems.

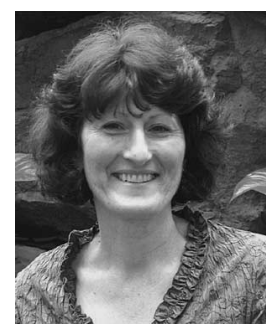

Marise Bafleur (M'95-SM'01) received the Engineer degree in solid state physics from the Institut National des Sciences Appliquées, Toulouse, France, in 1979, and the Ph.D. and State Doctorate degrees from Paul Sabatier University, Toulouse, in 1982 and 1987, respectively.

During her Ph.D. thesis from 1979 to 1982 , she worked on defect characterization of molecularbeam-epitaxy-grown GaAs layers. Since 1983, she has been with the Laboratoire d'Analyse et d'Architecture des Systèmes, Centre National de la Recherche Scientifique (LAAS-CNRS), Toulouse. From 1983 to 1987, she devoted her research activities to CMOS digital circuit design and modeling of its dynamic behavior. From 1987 to 1994, she oriented her activity toward smart-power IC design and technology. Then, she spent three years with Motorola, Phoenix, AZ, as, first, a Low-Power Designer and, second, working on TCAD predictive engineering. At the end of 1997, she came back to LAAS-CNRS Research Institute to lead a research activity in the field of electrostatic discharge (ESD) in CMOS and smart-power technologies. Since 2005, she has been managing the Power Management System Integration Research Group, LAAS-CNRS, which focuses its activities on the integration of passive devices and dc-dc converters, as well as on providing integrated ESD protection solutions for advanced CMOS and smart-power technologies. 


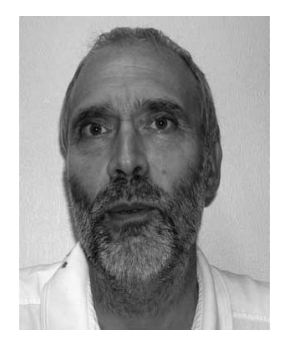

Philippe Perdu is currently a Senior Expert in microelectronics with the Centre National d'Etudes Spatiales (CNES), Toulouse, France. He has been leading the IC space application capability at CNES Laboratories (French National Space Agency) since 1988. He is strongly involved in the research and development of tools and methods for very large scale integration failure analysis.

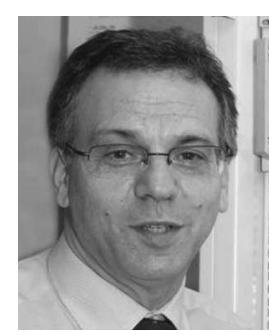

André Touboul is currently a Professor with the Université de Bordeaux 1, Talence, France, where was the Director of the Le Laboratoire d'Étude de l'Intégration des Composants et Systèmes Électroniques for eight years from 1998 to 2006 and is now with the Laboratoire de l'Intégration du Matériau au Systéme. He is currently involved in the physical modeling of failure mechanisms in a way to improve the reliability of integrated circuits and particularly of III-V transistors.

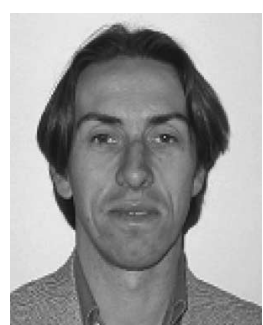

Dean Lewis was born in France in 1965. He received a degree in applied physics from the Ecole Normale Supérieure de Cachan, Cachan Cedex, France, in 1992, and the Ph.D. degree concerning thermal characterization of integrated circuits using a laser beam in 1996.

$\mathrm{He}$ is currently an Associate Professor with the Université de Bordeaux 1, Talence, France, where he teaches electronics and physics and is with the Laboratoire de l'Intégration du Matériau au Systéme. In 1997, he was with the Le Laboratoire d'Étude de l'Intégration des Composants et Systèmes Électroniques, Bordeaux, to develop new methodologies using a pulsed laser beam applied to integrated-circuit characterization. The first application concerns radiation-sensitivity evaluation with a pulsed laser of integrated circuits dedicated to space applications. The second application consists of developing laser frontside and backside techniques to evaluate the quality and reliability of integrated circuits. 